\title{
Interference Alignment under Training and Feedback Constraints
}

\author{
Baile Xie, Student Member, IEEE, Yang Li, Student Member, IEEE, \\ Hlaing Minn, Senior Member, IEEE, and Aria Nosratinia, Fellow, IEEE \\ The University of Texas at Dallas, Richardson, TX 75080, USA \\ Email: \{baile.xie, yang, hlaing.minn, aria\}@utdallas.edu
}

\begin{abstract}
We consider the effective degrees of freedom (DoF) achieved by interference alignment when channel state information (CSI) is acquired by training and feedback. For a flat blockfading $K \times K$ interference channel with power $P$ per transmitter and $M$ antennas per node, we show that interference alignment achieves higher DoF than orthogonal transmission (e.g., TDMA) only if the channel coherence time is large and the capacity of feedback link is at least as $\Theta(\log P)$. Under this condition, to maximize the effective $\mathrm{DoF}$, each receiver needs to feed back CSI via $\left(M^{2}-1\right) \log P$ bits per coherence interval; smaller growth rate of feedback bits will decrease the effective DoF. We also show that in the presence of training and feedback cost, $K=3$ achieves the optimal DoF for a broad range of channel characteristics; with larger number of user pairs, the DoF falls short of its optimum and beyond a certain point becomes a decreasing function of $K$.
\end{abstract}

Index Terms - Interference alignment, limited feedback, training, optimization, effective degrees of freedom

\section{INTRODUCTION}

Interference alignment is a class of promising transmission schemes that allows multiple pairs of transmitters and receivers to share the same spectrum. Each user pair can achieve half of the degrees of freedom (multiplexing gain) that can be achieved without interference [1]. For example, a $K \times K$ user interference network with $M$ antennas per user has a sum degrees of freedom (DoF) of $K M / 2$. To achieve the full DoF, the full channel state information (CSI) is required, with in general an infinite symbol extension.

Among the alignment schemes, spatial (signal space) interference alignment [2] requires no symbol extension (thus little delay) and is promising in practice [3]. A variety of schemes on spatial precoding design are proposed [4], [5], which require either full CSI or iterative approach with channels being reciprocal. Recently, Ayach et al. [6] considered that transmitters obtain CSI via analog feedback, while Thukral et al. [7] and Krishnamachari et al. [8] applied limited feedback [9] for CSI exchange. For limited feedback, it had shown that if the number of feedback bits grows logarithmically with signal-to-noise-ratio (SNR), the full DoF is sustained in constant (non-fading) channels.

In this paper, we study the effective DoF achieved by interference alignment when both training [10] and limited feedback [9] are taken into consideration. We have found both lower and upper bounds of ergodic sum DoF for block-fading channels. It is shown that at high SNR, interference alignment outperforms orthogonal transmission schemes (e.g., TDMA) when the channel coherence time is large and the capacity of the feedback link scales at least $\Theta(\log P)$. In this case, feeding back $\left(M^{2}-1\right) \log P$ bits from each receiver achieves the optimal DoF, given alignment is feasible [2]. We have also found that in many practical scenarios, a small number of user pairs suffices to achieve the optimal effective DoF. For example, $K=3$ user pairs maximize the effective DoF for a coherence interval ranging from 150 to 600 symbols and the number of antennas $M$ from 2 to 8 ; with higher number of user pairs, the effective DoF becomes a decreasing function of $K$.

Notations: Throughout the paper, the boldface low-case letter a stands for vector while upper-case $\mathbf{A}$ represents matrix. $\mathbf{A}^{\dagger}$ indicates the Hermitian transpose of $\mathbf{A}$. $\|\mathbf{a}\|$ means $\ell_{2}$-norm while $\|\mathbf{A}\|_{F}$ refers to Frobenius norm. $\mathcal{C N}(\mathbf{a}, \mathbf{A})$ is complex Gaussian distribution with mean a and covariance matrix $\mathbf{A}$. Inv- $\chi^{2}(a)$ is inverse $\chi^{2}$ distribution with degree of freedom $a$. $\mathbf{E}[\cdot]$ stands for expectation. $\mathbb{C}^{M \times N}$ is the $M \times N$ dimension complex space. $\log (\cdot)$ is base $2 \log$ arithm. $\lfloor\cdot\rfloor$ and $\lceil\cdot\rceil$ represent floor and ceiling operation, respectively. For some positive $c_{1}$ and $c_{2}$, and sufficiently large $x$ :

$$
\begin{aligned}
f(x) & =O(g(x)): & & |f(x)|<c_{1}|g(x)| \\
f(x) & =\Theta(g(x)): & c_{2}|g(x)|<|f(x)| & <c_{1}|g(x)|
\end{aligned}
$$

The rest of the paper is organized as follows. Section II describes system model. In Section III we outline our approach by taking training and feedback into consideration. Section IV formulates the effective DoF and analyzes its lower bound and upper bound. Discussions and simulation results on the requirement of feedback bits and coherence interval are included in this section as well. In Section V, the optimal number of user pairs is investigated. Finally, Section VI concludes this paper.

\section{SYSTEM MODEL}

A $K \times K$ narrowband interference channel is considered where transmitters and receivers are equipped with $M$ antennas each. The signal at receiver $k$ is:

$$
\mathbf{y}_{k}=\sum_{\ell=1}^{K} \mathbf{H}_{k \ell} \mathbf{x}_{\ell}+\mathbf{z}_{k}, \quad \text { for } k=1,2 \cdots, K
$$


where $\mathbf{x}_{\ell} \in \mathbb{C}^{M \times 1}$ is the signal from transmitter $\ell$ and $\mathbf{H}_{k \ell} \in \mathbb{C}^{M \times M}$ is the channel coefficient matrix between the transmitter $\ell$ and the receiver $k$. In the above, $\mathbf{z}_{k} \in \mathbb{C}^{M \times 1}$ is additive noise with distribution $\mathcal{C N}\left(\mathbf{0}, \sigma^{2} \boldsymbol{I}\right)$. In this paper, we consider a block-fading model where the channel coefficients remain constant for $T$-symbols (a block). Each entity of $\mathbf{H}_{k \ell}$ is i.i.d. $\mathcal{C N}(0,1)$.

In interference alignment, the transmitter $\ell$ sends $d_{\ell}$ data streams (spatial multiplexing) simultaneously; more precisely:

$$
\mathbf{x}_{\ell}=\sum_{p=1}^{d_{\ell}} \mathbf{v}_{\ell}^{p} s_{\ell}^{p}
$$

where $\mathbf{v}_{\ell}^{p} \in \mathbb{C}^{M \times 1}$ is the unit-norm precoding vector associated with $s_{\ell}^{p}$, the data stream $p$. Each transmitter has the total power constraint $P$, which is equally allocated to their data streams, i.e., $\mathbf{E}\left[\left\|s_{\ell}^{p}\right\|^{2}\right]=P / d_{\ell}$.

At the receiver $k$, a receiving vector $\mathbf{u}_{k}^{q} \in \mathbb{C}^{M \times 1}$ for data stream $q$ is applied to suppress the interference from other streams:

$$
\begin{aligned}
\left(\mathbf{u}_{k}^{q}\right)^{\dagger} \mathbf{y}_{k} & =\left(\mathbf{u}_{k}^{q}\right)^{\dagger} \mathbf{H}_{k k} \mathbf{v}_{k}^{q} s_{k}^{q}+\left(\mathbf{u}_{k}^{q}\right)^{\dagger} \sum_{p=1, p \neq q}^{d_{k}} \mathbf{H}_{k k} \mathbf{v}_{k}^{p} s_{k}^{p} \\
& +\left(\mathbf{u}_{k}^{q}\right)^{\dagger} \sum_{\ell=1, \ell \neq k}^{K} \sum_{p=1}^{d_{\ell}} \mathbf{H}_{k \ell} \mathbf{v}_{\ell}^{p} s_{\ell}^{p}+\left(\mathbf{u}_{k}^{q}\right)^{\dagger} \mathbf{z}_{k} .
\end{aligned}
$$

With full CSI, the precoding and receiving vectors in interference alignment should satisfy

$$
\begin{aligned}
\left(\mathbf{u}_{k}^{q}\right)^{\dagger} \mathbf{H}_{k \ell} \mathbf{v}_{\ell}^{p} & =0, \forall \ell \neq k \\
\left(\mathbf{u}_{k}^{q}\right)^{\dagger} \mathbf{H}_{k k} \mathbf{v}_{k}^{p} & =0, \forall q \neq p \\
\left|\left(\mathbf{u}_{k}^{q}\right)^{\dagger} \mathbf{H}_{k k} \mathbf{v}_{k}^{q}\right| & >0, \forall k, q .
\end{aligned}
$$

The specific designs of the precoding and receiving vectors can be found in, e.g., [4], [5].

\section{INTERFERENCE ALIGNMENT With TRAINING AND FEEDBACK}

In this paper, training [10] and feedback [9] are used to acquire and exchange CSI which is required for interference alignment. Specifically, it consists of the following steps.

1) Forward Link Training: All transmit antennas send a pilot symbol in a time-division manner over $K \times M$ timeslots (symbols) [10], [11]. Each receiver estimates their channels from all the transmitters. For simplicity, we assume the channels are estimated perfectly.

2) Channel Quantization: After obtaining CSI at the receiver $k$, it quantizes $\mathbf{H}_{k \ell}$ into $N_{f}$ bits [9], for $\ell=1, \cdots, K$, resulting in a total of $N_{f} K$ quantization bits at one receiver. More precisely, $\mathbf{H}_{k \ell}$ is expanded into a vector $\mathbf{h}_{k \ell}=$ $\operatorname{vec}\left(\mathbf{H}_{k \ell}\right) \in \mathbb{C}^{M^{2} \times 1}$, and the direction of $\mathbf{h}_{k \ell}$, i.e., $\tilde{\mathbf{h}}_{k \ell}=$ $\mathbf{h}_{k \ell} /\left\|\mathbf{h}_{k \ell}\right\|$ is quantized according to a quantization codebook $\mathcal{C}=\left\{\mathbf{w}_{i}: i=1, \cdots, 2^{N_{f}}\right\}[9]$ as:

$$
\hat{\mathbf{h}}_{k l} \triangleq \arg \max _{\mathbf{w}_{i} \in \mathcal{C}}\left|\tilde{\mathbf{h}}_{k \ell}^{\dagger} \mathbf{w}_{i}\right|
$$

where $\mathbf{w}_{i}$ is of unit-norm.
3) Channel Feedback: We assume a shared feedback channel that allows $B_{f}$ feedback bits per symbol duration (of forward link) to be received simultaneously by all the transmitters. The receivers feed back the index of the chosen codeword $\hat{\mathbf{h}}_{k \ell}$ via the feedback channel sequentially. In order for the transmitters to obtain the quantized version of all channels, feedback overhead is equivalent to $K^{2} N_{f} /\left(c B_{f}\right)^{1}$ symbol durations of the forward link, where $c$ is the code rate of the error correcting code for feedback bits.

In addition, depending on the infrastructure of the whole system, unless the reverse channel is already known at the transmitter, another $K M$ symbols are required for training of the reverse link, assuming the same training process is used as the forward link. Consequently, the total time slots spent on training is $\tau K M$, where $\tau=1$ or 2 depends on whether reverse training is required or not.

4) Interference Alignment with Quantized CSI: The transmitters reshape $\hat{\mathbf{h}}_{k \ell}$ to form a quantized channel $\hat{\mathbf{H}}_{k \ell}$ for precoding design. The precoding vectors $\left\{\hat{\mathbf{v}}_{k}^{q}\right\}$ are calculated by regarding $\hat{\mathbf{H}}_{k \ell}$ as the true channel, ${ }^{2}$ for $k=1, \cdots, K$ and $q=1, \cdots, d_{k}$, yielding:

$$
\begin{aligned}
\left(\hat{\mathbf{u}}_{k}^{q}\right)^{\dagger} \hat{\mathbf{H}}_{k \ell} \hat{\mathbf{v}}_{\ell}^{p} & =0, \forall \ell \neq k \\
\left(\hat{\mathbf{u}}_{k}^{q}\right)^{\dagger} \hat{\mathbf{H}}_{k k} \hat{\mathbf{v}}_{k}^{p} & =0, \forall q \neq p \\
\left|\left(\hat{\mathbf{u}}_{k}^{q}\right)^{\dagger} \hat{\mathbf{H}}_{k k} \hat{\mathbf{v}}_{k}^{q}\right| & >0, \forall k, q
\end{aligned}
$$

where $\left\{\hat{\mathbf{u}}_{k}^{q}\right\}$ is the receiving vector for the data stream $q$ at the receiver $k$. The data stream $q$ is decoded by correlating $\mathbf{y}_{k}$ with $\hat{\mathbf{u}}_{k}^{q}$ to suppress the interference from all other data streams (from the same and different transmitters). Thus we have

$$
\begin{aligned}
\left(\hat{\mathbf{u}}_{k}^{q}\right)^{\dagger} \mathbf{y}_{k} & =\left(\hat{\mathbf{u}}_{k}^{q}\right)^{\dagger} \mathbf{H}_{k k} \hat{\mathbf{v}}_{k}^{q} s_{k}^{q}+\left(\hat{\mathbf{u}}_{k}^{q}\right)^{\dagger} \sum_{p=1, p \neq q}^{d_{k}} \mathbf{H}_{k k} \hat{\mathbf{v}}_{k}^{p} s_{k}^{p} \\
& +\left(\hat{\mathbf{u}}_{k}^{q}\right)^{\dagger} \sum_{\ell=1, \ell \neq k}^{K} \sum_{p=1}^{d_{\ell}} \mathbf{H}_{k \ell} \hat{\mathbf{v}}_{\ell}^{p} s_{\ell}^{p}+\left(\hat{\mathbf{u}}_{k}^{q}\right)^{\dagger} \mathbf{z}_{k} .
\end{aligned}
$$

Therefore, the rate of data stream $q$ at the receiver $k$ is

$$
R_{k}^{q}=\log \left(1+\frac{\frac{P}{d_{k}}\left\|\left(\hat{\mathbf{u}}_{k}^{q}\right)^{\dagger} \mathbf{H}_{k k} \hat{\mathbf{v}}_{k}^{q}\right\|^{2}}{I_{k, 1}+I_{k, 2}+\sigma^{2}}\right)
$$

where

$$
\begin{aligned}
& I_{k, 1}=\frac{P}{d_{k}} \sum_{p=1, p \neq q}^{d_{k}}\left\|\left(\hat{\mathbf{u}}_{k}^{q}\right)^{\dagger} \mathbf{H}_{k k} \hat{\mathbf{v}}_{k}^{p}\right\|^{2} \\
& I_{k, 2}=\sum_{l=1, l \neq k}^{K} \sum_{p=1}^{d_{l}} \frac{P}{d_{l}}\left\|\left(\hat{\mathbf{u}}_{k}^{q}\right)^{\dagger} \mathbf{H}_{k l} \hat{\mathbf{v}}_{l}^{p}\right\|^{2} .
\end{aligned}
$$

Note that $I_{k, 1}$ is the intra-user interference from the transmitter $k$ and $I_{k, 2}$ is the inter-user interference from other transmitters.

\footnotetext{
${ }^{1}$ Some beam design algorithms (such as minimum interference leakage algorithm in [4]) do not require the knowledge of the direct channel ( $\left.\left\{\mathbf{H}_{k k}\right\}\right)$, resulting a feedback overhead of $K(K-1) N_{f} /\left(c B_{f}\right)$ symbol durations.

${ }^{2}$ Whether or not any other alignment scheme exists, rather than this naive interference alignment, which achieves higher DoF, is still unknown.
} 


\section{EFFECTIVE DoF of INTERFERENCE ALIGNMENT}

We analyze the sum DoF achieved by interference alignment under practical scenarios where the resource used to obtain CSI is considered. In this case, the channel coherence time and the capacity of the feedback link have significant impacts on the effective DoF as well as the optimal feedback strategy. On one hand, the receivers may quantize their channels with higher precision (larger $N_{f}$ ), which will intuitively lead to smaller interference (due to channel quantization error) at the receivers. Nevertheless, this also requires more system resource thus reducing the resource available for data transmission. On the other hand, the receivers may use fewer feedback bits so that more resources are available for data transmission, though the interference at the receivers is also increased. We aim to characterize the optimal choice of $N_{f}$.

Taking into consideration of training and feedback described in Section III, the normalized effective sum rate is

$$
\mathcal{R} \triangleq\left(1-\tau \frac{K M}{T}-\frac{K^{2} N_{f}}{c T B_{f}}\right) \sum_{k=1}^{K} \sum_{q=1}^{d_{k}} \mathbf{E}\left[R_{k}^{q}\right]
$$

where $B_{f}$ is the capacity (bits per forward link symbol) of the feedback link. The effective DoF (DoF) is therefore

$$
\mathcal{D}=\lim _{P \rightarrow \infty} \frac{\mathcal{R}}{\log P}
$$

Previous results had shown that $N_{f}$ should grow linearly with $\log P$ to limit the interference power [12], [7], [8]. In this paper, we consider $N_{f}$ as a more general function of $P$, denoted as $N_{f}(P)$.

\section{A. Lower Bound}

For brevity, we only focus on one term, e.g., $\mathbf{E}\left[R_{k}^{q}\right]$, in the summation in (15); the analyses of other terms are the same.

First, we rewrite

$$
\left(\hat{\mathbf{u}}_{k}^{q}\right)^{\dagger} \mathbf{H}_{k \ell} \hat{\mathbf{v}}_{\ell}^{p}=\left\langle\mathbf{h}_{k \ell}, \mathbf{b}_{k, \ell}^{q, p}\right\rangle=\left\|\mathbf{h}_{k \ell}\right\| \tilde{\mathbf{h}}_{k \ell}^{\dagger} \mathbf{b}_{k, \ell}^{q, p}
$$

where the $((m-1) M+\ell)$ th element of $\mathbf{b}_{k, \ell}^{q, p} \in \mathbb{C}^{M^{2} \times 1}$ is given by $\hat{\mathbf{u}}_{k}^{q}(\ell) \times \hat{\mathbf{v}}_{\ell}^{p}(m)$, respectively. Therefore, based on the results in [7], [8],

$$
I_{k, 1}=\sum_{p=1, p \neq q}^{d_{k}} \frac{P}{d_{k}}\left\|\mathbf{h}_{k k}\right\|^{2}\left\|\tilde{\mathbf{h}}_{k k}^{\dagger} \mathbf{b}_{k, k}^{q, p}\right\|^{2} \leq O\left(P 2^{-\frac{N_{f}(P)}{M^{2}-1}}\right) .
$$

Similarly,

$$
I_{k, 2} \leq O\left(P 2^{-\frac{N_{f}(P)}{M^{2}-1}}\right) .
$$

From (12), we have:

$$
\mathbf{E}\left[R_{k}^{q}\right]>\mathbf{E}\left[\log \left(\frac{\frac{P}{d_{k}}\left\|\left(\hat{\mathbf{u}}_{k}^{q}\right)^{\dagger} \mathbf{H}_{k k} \hat{\mathbf{v}}_{k}^{q}\right\|^{2}}{I_{k, 1}+I_{k, 2}+\sigma^{2}}\right)\right] \triangleq R_{\text {low }}
$$

Substituting (18) and (19) into (20) gives

$$
R_{\text {low }} \geq \mathbf{E}\left[\log \left(\frac{\frac{P}{d_{k}}\left\|\left(\hat{\mathbf{u}}_{k}^{q}\right)^{\dagger} \mathbf{H}_{k k} \hat{\mathbf{v}}_{k}^{q}\right\|^{2}}{O\left(P 2^{\frac{-N_{f}(P)}{M^{2}-1}}\right)+\sigma^{2}}\right)\right] .
$$

Now, we consider the order of interference power $P 2^{\frac{-N_{f}(P)}{M^{2}-1}}=P^{\alpha}$. Naturally, $\alpha$ is less than or equal to 1 (with equality indicating a constant number of feedback bits). On the other hand, it is unnecessary to make $\alpha<0$, in which case the noise dominates and the whole system is noise-limited rather than interference limited. We focus on $\alpha=0$ and $0<\alpha \leq 1$ in the following.

1) $\alpha=0$ : In this case, $N_{f}(P)=\left(M^{2}-1\right) \log P$. The interference power $2^{-\frac{N_{f}(P)}{M^{2}-1}} P$ is bounded as $P$ goes to infinity, and the whole denominator in (21) approaches a constant as $P$ grows. We have

$$
\begin{aligned}
R_{\text {low }} & \approx \mathbf{E}\left[\log \left(P f_{1}(\mathbf{H})\right)\right] \\
& \approx \log P+O(1)
\end{aligned}
$$

where $f_{1}(\mathbf{H})$ is a function dependent on channel statistics but independent of $P$.

2) $0<\alpha \leq 1$ : We have $N_{f}(P)=(1-\alpha)\left(M^{2}-1\right) \log P$, and the noise power $\sigma^{2}$ in (21), compared with $P^{\alpha}$, becomes negligible at high SNR. Thus

$$
\begin{aligned}
R_{\text {low }} & \approx \mathbf{E}\left[\log \left(f_{2}(\mathbf{H}) P^{1-\alpha}\right)\right] \\
& =(1-\alpha) \log P+O(1)
\end{aligned}
$$

where $f_{2}(\mathbf{H})$ is also independent of $P$.

\section{B. Upper Bound}

Define $\Delta \mathbf{H}_{k \ell}=\mathbf{H}_{k \ell} /\left\|\mathbf{H}_{k \ell}\right\|_{F}-\hat{\mathbf{H}}_{k \ell}$ and $\Delta \mathbf{h}_{k \ell}=$ $\operatorname{vec}\left(\Delta \mathbf{H}_{k \ell}\right)$. From (8) and (9), we have

$$
\begin{aligned}
& I_{k, 1}=\frac{P}{d_{k}} \sum_{p=1, p \neq q}^{d_{k}}\left\|\mathbf{H}_{k k}\right\|_{F}^{2}\left\|\left(\hat{\mathbf{u}}_{k}^{q}\right)^{\dagger} \Delta \mathbf{H}_{k k} \hat{\mathbf{v}}_{k}^{p}\right\|^{2}, \\
& I_{k, 2}=\sum_{\ell=1, \ell \neq k}^{K} \sum_{p=1}^{d_{\ell}} \frac{P}{d_{\ell}}\left\|\mathbf{H}_{k \ell}\right\|_{F}^{2}\left\|\left(\hat{\mathbf{u}}_{k}^{q}\right)^{\dagger} \Delta \mathbf{H}_{k \ell} \hat{\mathbf{v}}_{\ell}^{p}\right\|^{2} .
\end{aligned}
$$

By the Jensen inequality, we have

$$
\begin{aligned}
& \mathbf{E}\left[R_{k}^{q}\right] \leq \log \left(1+\mathbf{E}\left[\frac{\frac{P}{d_{k}}\left\|\left(\hat{\mathbf{u}}_{k}^{q}\right)^{\dagger} \mathbf{H}_{k k} \hat{\mathbf{v}}_{k}^{q}\right\|^{2}}{I_{k, 1}+I_{k, 2}+\sigma^{2}}\right]\right) \\
& \stackrel{(A)}{<} \log \left(1+\mathbf{E}\left[\frac{d_{\ell} \lambda_{\max }^{2}\left(\mathbf{H}_{k k}\right)}{d_{k}\left\|\mathbf{H}_{k \ell}\right\|_{F}^{2}\left\|\left(\hat{\mathbf{u}}_{k}^{q}\right)^{\dagger} \Delta \mathbf{H}_{k \ell} \hat{\mathbf{v}}_{\ell}^{p}\right\|^{2}}\right]\right) \\
& \stackrel{(B)}{=} \log \left(1+\frac{d_{\ell}}{d_{k}} \mathbf{E}\left[\lambda_{\max }^{2}\left(\mathbf{H}_{k k}\right)\right] \mathbf{E}[\eta]\right)
\end{aligned}
$$

where $\lambda_{\max }\left(\mathbf{H}_{k k}\right)$ is the largest eigenvalue of $\mathbf{H}_{k k}$ and $\eta=\left\|\mathbf{H}_{k \ell}\right\|_{F}^{-2}\left\|\Delta \mathbf{h}_{k \ell}^{\dagger} \mathbf{b}_{k, \ell}^{q, p}\right\|^{-2}$. The step (A) holds because of discarding non-negative terms $I_{k, 1}, \sigma^{2}$ and other terms in $I_{k, 2}$. The step (B) follows from the independence between $\mathbf{H}_{k k}$ and $\mathbf{H}_{k \ell}, k \neq \ell$. The normalized quantization error

$$
\Delta \tilde{\mathbf{w}}_{k \ell}=2^{N_{f}(P) / 2\left(M^{2}-1\right)}\left\|\Delta \mathbf{h}_{k \ell}\right\|
$$


has an asymptotic probability density function (pdf) that is independent of $N_{f}$ [13]. Due to the independence between the norm and angle of $\operatorname{vec}\left(\mathbf{H}_{k \ell}\right)$, we have [14]

$$
\begin{aligned}
\mathbf{E}[\eta] & =2^{\frac{N_{f}(P)}{M^{2}-1}} \mathbf{E}\left[\left\|\mathbf{H}_{k \ell}\right\|_{F}^{-2}\right] \mathbf{E}\left[\left\|\Delta \tilde{\mathbf{w}}_{k \ell}^{\dagger} \mathbf{b}_{k, \ell}^{q, p}\right\|^{-2}\right] \\
& =2^{\frac{N_{f}(P)}{M^{2}-1}} \times \frac{\mu}{M^{2}-1}
\end{aligned}
$$

where $\left\|\mathbf{H}_{k \ell}\right\|_{F}^{-2} / 2 \sim \operatorname{Inv}-\chi^{2}\left(2 M^{2}\right)$ with mean $1 /\left(2 M^{2}-2\right)$ and $\mu=\mathbf{E}\left[\left\|\Delta \tilde{\mathbf{w}}_{k \ell}^{\dagger} \mathbf{b}_{k, \ell}^{q, p}\right\|^{-2}\right]$ is a constant. Substituting (28) into (26), we have

$$
\begin{aligned}
\mathbf{E}\left[R_{k}^{q}\right] & <\log \left(\Theta\left(2^{\frac{N_{f}(P)}{M^{2}-1}}\right)\right) \\
& =(1-\alpha) \log P+O(1) \triangleq R_{\mathrm{up}} .
\end{aligned}
$$

The upper bound holds for both $\alpha=0$ and $0<\alpha \leq 1$.

\section{Effective DoF}

Note that for either $\alpha=0$ or $0<\alpha \leq 1$, the lower and upper bounds coincide except for a constant (independent of $P$ ). Therefore, the ergodic rate of the data stream $q$ at the pair $k$ can be written as

$$
\mathbf{E}\left[R_{k}^{q}\right] \approx(1-\alpha) \log P+O(1)
$$

in both cases. In the following, we analyze the effective throughput using this general form.

From (15), $B_{f}$ should be at least on the same order of $N_{f}=\Theta(\log P)$; otherwise, it is impossible to feed back the quantized CSI within a coherence block. For purpose of illustration, we write $B_{f}=\beta \log P$. Substituting (30) and $B_{f}$ into (16), then we have

$$
\mathcal{D}=\left(1-\tau \frac{K M}{T}-\frac{K^{2}(1-\alpha)\left(M^{2}-1\right)}{c \beta T}\right)(1-\alpha) \sum_{k=1}^{K} d_{k} .
$$

Due to the symmetry of all user pairs, $d_{k}=d$ for our consideration of spatial interference alignment. From [2, Theorem 1], we have the feasibility requirement

$$
2 M-(K+1) d \geq 0 .
$$

Thus, the effective DoF writes as

$$
\begin{aligned}
\mathcal{D}= & \left(1-\tau \frac{K M}{T}-\frac{K^{2}(1-\alpha)\left(M^{2}-1\right)}{c \beta T}\right) \\
& \times\left\lfloor\frac{2 M}{K+1}\right\rfloor(1-\alpha) K .
\end{aligned}
$$

Note that (33) is a quadratic function of $(1-\alpha)$; the maxima of the function is achieved when

$$
(1-\alpha)^{\star}=c \beta(T-\tau K M) /\left(2 K^{2}\left(M^{2}-1\right)\right) .
$$

The corresponding $\mathcal{D}$ is

$$
\mathcal{D}^{\star}=\left(1-\tau \frac{K M}{T}\right)\left(\frac{K}{2}\right)\left\lfloor\frac{2 M}{K+1}\right\rfloor(1-\alpha)^{\star} .
$$

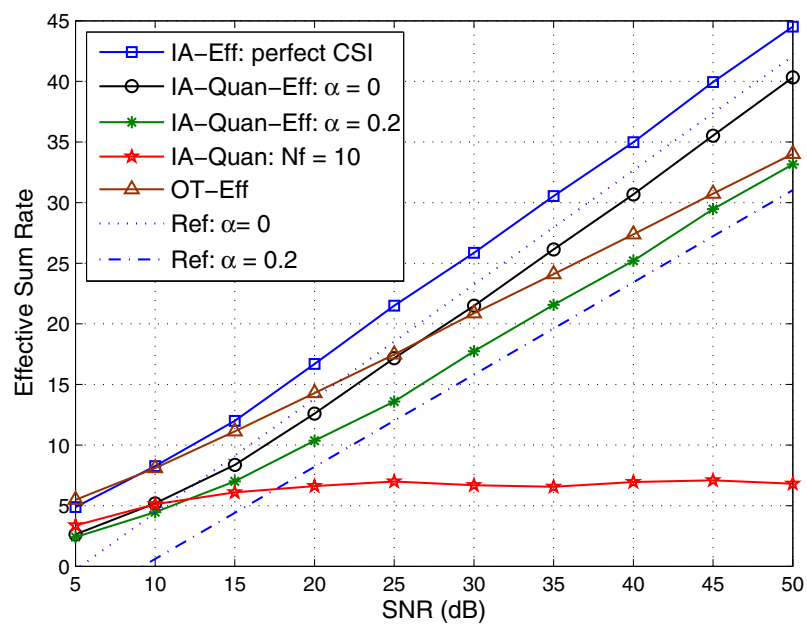

Figure 1. Comparison of effective sum rate: $K=3, M=2, T=600, \beta=$ 1 , IA $=$ interference alignment, OT $=$ orthogonal transmission

Recall that the effective DoF of the orthogonal transmission is [10]

$$
\mathcal{D}_{\mathrm{OT}}=M(1-M / T)
$$

In order for interference alignment to achieve a larger DoF than the orthogonal transmission, it requires that

$$
(1-\alpha)^{\star} \geq \frac{(T-M)(K+1)}{(T-\tau K M) K}>1
$$

which is out of the domain $0 \leq(1-\alpha) \leq 1$. Because of the quadratic property of $\mathcal{D}$ in (33), the optimal value of $(1-\alpha)$ should be 1 , namely, $\alpha=0$. Hence, large feedback $N_{f}(P)=$ $\left(M^{2}-1\right) \log P$ maximizes the effective DoF.

To verify the above results, consider a numerical example where $T=600,{ }^{3} c=\tau=\beta=1$ and $K=3, M=2$. The simulated effective sum rates versus SNR are plotted in Fig. 1, where random vector quantization [15] is employed for CSI feedback with $N_{f}=\left\lceil(1-\alpha)\left(M^{2}-1\right) \log (\mathrm{SNR})\right\rceil$ bits. At high SNR, the slopes of the simulated sum rates are almost identical to that of the analytical result (33) (see the reference lines) for both $\alpha=0$ and 0.2 . With large feedback bits $(\alpha=0)$, only a constant loss exists in the effective sum rate with limited feedback, compared to that with genie-aided CSI, which indicates that $\left(M^{2}-1\right) \log P$ bits of CSI feedback preserves DoF. Furthermore, the results also imply that $\alpha=0$ is optimal: for $\alpha=0.2$, the sum rate has smaller DoF than $\alpha=0$, though it still achieves higher DoF than orthogonal transmission. Finally, if the number of feedback bits is fixed, e.g., $N_{f}=10$, the effective sum rate does not increase with SNR and the system becomes interference limited.

Now, we characterize the minimum requirement of coherence interval to perform interference alignment. Since $\alpha=0$

\footnotetext{
${ }^{3}$ This corresponds to typical scenarios in LTE where a mobile operates at $2.1 \mathrm{GHz}$ with speed $5 \mathrm{~km} / \mathrm{h}$, for a symbol rate of $15 \mathrm{kHz}$.
} 
is optimal, we concentrate on this case throughout the rest of the discussion; from (33):

$$
\mathcal{D}=\left(1-\tau \frac{K M}{T}-\frac{K^{2}\left(M^{2}-1\right)}{c \beta T}\right)\left\lfloor\frac{2 M}{K+1}\right\rfloor K .
$$

From (38) and (36), in order for $\mathcal{D}>\mathcal{D}_{\mathrm{OT}}, T$ should satisfy:

$$
T>d_{k}\left(\tau K^{2} M+\frac{K^{3}\left(M^{2}-1\right)}{c \beta}-M^{2}\right) /\left(K d_{k}-M\right)
$$

where $d_{k}=\left\lfloor\frac{2 M}{K+1}\right\rfloor$. Eq. (39) offers a guideline on determining when to favor interference alignment over orthogonal transmissions. If $T$ is small, the overhead (due to training and feedback) of interference alignment will counteract the gain over orthogonal transmissions, or even result in throughput loss. If $T$ is large enough (static channels), interference alignment will attain higher DoF than orthogonal schemes. Intuitively, overhead is negligible for large $T$, and in this case the alignment is almost equivalent to that with perfect CSI, which is known to outperform orthogonal transmission.

\section{Optimal Number of CoOperative User Pairs}

With priori CSI, the sum DoF achieved by interference alignment grows with the number of user pairs $K$ and antennas $M$ [1], however, once considering the resource to obtain CSI, the effective sum DoF may not increase as $K$ and $M$ grow. For example, given capacity of the feedback link, coherence interval and $M$, we can select fewer pairs to collaborate in the alignment, requiring smaller overhead (due to training and feedback), and thus leaves more resource for data transmission. But since a smaller number of user pairs are active, the overall throughput may be reduced. Another choice will be to select more user pairs to collaborate, however, it requires larger overhead and may limit the resource for data transmission. Thereby, an optimal choice of cooperative pairs $K$ (and $M$ ) shall exist, which is further shown to depend on the capacity of the feedback link and the channel coherence time.

We first inspect the effective DoF given by (38); the overhead term

$$
\left(1-\tau \frac{K M}{T}-\frac{K^{2}\left(M^{2}-1\right)}{c \beta T}\right)
$$

is a monotonically decreasing function of $K$, which indicates that the more cooperative user pairs, the more training and feedback overhead is required. Indeed, in term of overhead cost, $M$ has the same impact as $K$, which is easily seen from (40). Then, we note that the DoF term

$$
\left\lfloor\frac{2 M}{K+1}\right\rfloor K
$$

increases ${ }^{4}$ with $K$. Therefore, it is desirable to find $K$ to maximize the effective DoF (the product of two terms).

\begin{tabular}{|c|c|c|c|c|c|c|c|c|c|}
\hline & \multicolumn{7}{|c|}{ Number of Antennas $M$} & \multirow{2}{*}{$T$ (symbols) } & \multirow[b]{2}{*}{$\beta$} \\
\hline & 2 & 3 & 4 & 5 & 6 & 7 & 8 & & \\
\hline \multirow{4}{*}{$K_{\text {opt }}$} & 3 & 3 & 3 & 3 & 3 & 3 & 3 & 150 & \multirow{2}{*}{1} \\
\hline & 3 & 5 & 3 & 3 & 3 & 3 & 3 & 600 & \\
\hline & 3 & 3 & 3 & 3 & 3 & 3 & 3 & 150 & \multirow{2}{*}{2} \\
\hline & 3 & 5 & 3 & 4 & 3 & 3 & 3 & 600 & \\
\hline
\end{tabular}

\footnotetext{
${ }^{4}$ Due to the integer constraint, it is not a strictly increasing function of $K$.
}

Table I

OPTIMAL NUMBER OF USER PAIRS

Note that for given $M$, from (32), $K$ could take up to $\left\lfloor\frac{2 M}{d}\right\rfloor-1$, for $d=1, \cdots,\lfloor M / 2\rfloor$, where $d$ is the desired DoF per user pair. The optimal $K$ is

$$
K_{\mathrm{opt}}=\arg \max _{3 \leq K \leq 2 M-1} \mathcal{D} \text {. }
$$

In order to evaluate the above optimization problem, we consider several practical scenarios where $T$ and $\beta$ take different values. Specifically, we consider again $T=600$, the same as the previous numerical example in Section IV-C, and $T=150$ that corresponds to a mobile speed of $20 \mathrm{~km} / \mathrm{h}$. Also, different capacities of the feedback link are considered, where $\beta=1$ and 2 , respectively. Finally, we allow up to $M=8$ antennas per user, which is the maximum antenna number supported by present systems.

The optimal value of $K$ is found via (42) under various system parameters, and the results are listed in Table. I. For most cases, interference alignment achieves the maximum effective DoF with 3 user pairs; a large $K$ may even reduce the effective DoF. Intuitively, for many practical cases, the cost of training and feedback is significant and thus it is optimal to allow only a small number of user pairs to cooperate.

To further illustrate the relation among $K, M$ and $\mathcal{D}$, we plot the effective DoF associated with Table I in Fig. $2 .{ }^{5}$ For $T=150$ and $\beta=1, \mathcal{D}$ even decreases with $M$ : in order to obtain CSI of the additional antennas, more feedback bits are required which significantly reduces the data transmission time, which leads to smaller effective DoF in the presence of short coherence time and small feedback capacity. In this case, the effective DoF of interference alignment is even less than that of orthogonal transmission schemes. As $T$ and $\beta$ increase, e.g., $T=600$ and $\beta=2$, using more antennas produces larger effective DoF due to less significant training and feedback cost. However, as $M$ continues to increase, the DoF attained by interference alignment becomes close to that of orthogonal schemes or even worse since the overhead cost keeps growing as well. Therefore, interference alignment produces DoF gains in systems with small $M$ but with large coherence time $T$ and feedback capacity $B_{f}$.

To obtain some intuitions, we consider two limiting cases. With increasing channel stability, the overhead with training and feedback becomes negligible; as $T$ goes to infinity, e.g., constant channel, the whole discussion falls back into the original interference alignment with genie-aided CSI. In this case, activating as many users as possible is the optimal strategy.

\footnotetext{
${ }^{5}$ The unsmoothness of the plots is due to the integer constraints of DoF and $K$, i.e., the floor operation in (41).
} 


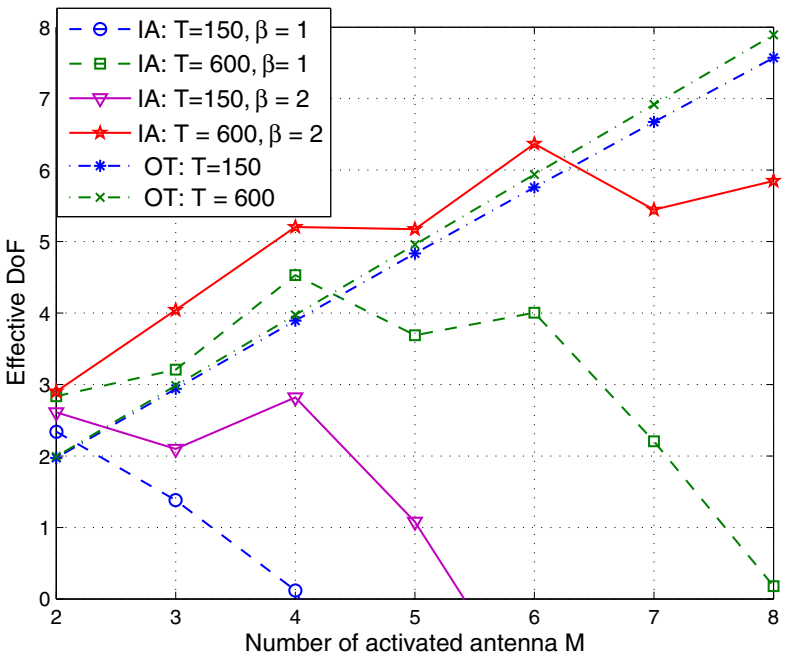

Figure 2. Effective DoF versus the number of antennas with $K_{\mathrm{opt}}$

Now, we consider $B_{f}$ goes to infinity, i.e., the receivers can feed back CSI with infinite preciseness and zero delay; the effective degrees of freedom is

$$
\mathcal{D}^{\infty}=\left(1-\tau \frac{K M}{T}\right)\left\lfloor\frac{2 M}{K+1}\right\rfloor K
$$

The optimal number of cooperative pairs is therefore

$$
K_{\mathrm{opt}}^{\infty}=\arg \max _{3 \leq K \leq 2 M-1} \mathcal{D}^{\infty}
$$

One can verify that, $K_{\mathrm{opt}}^{\infty}$ is up to 9 for $T=150$ and 11 for $T=600$ (with the same parameter settings as previous analysis), which serves as an upper bound on the number of users.

\section{CONCLUSION}

In this work, we have shown, both analytically and numerically, that the channel coherence time and the capacity of feedback link are critical for spatial interference alignment when taking the training and feedback into consideration. Given sufficient coherence interval and feedback capacity, the optimal effective DoF is achieved if each receiver feeds back $\left(M^{2}-1\right) \log P$ bits of CSI to the transmitters; insufficient feedback bits might result in even a smaller DoF than that of orthogonal transmission schemes. For many practical scenarios, we have found that a small number of user pairs suffices to achieve the optimal effective DoF, and it is unnecessary or suboptimal to include large number of user pairs to cooperate.

\section{ACKNOWLEDGMENT}

This work is supported in part by a gift money from RIM. The second author greatly appreciates the discussions with Chenwei Wang from University of California, Irvine.

\section{REFERENCES}

[1] V. Cadambe and S. Jafar, "Interference alignment and degrees of freedom of the k-user interference channel," IEEE Trans. Inf. Theory, vol. 54, no. 8, pp. $3425-3441,2008$.

[2] C. Yetis, T. Gou, S. Jafar, and A. Kayran, "On feasibility of interference alignment in mimo interference networks," IEEE Trans. Signal Process., vol. 58 , no. 9 , pp. $4771-4782,2010$.

[3] C. Suh, M. Ho, and D. Tse, "Downlink interference alignment," http: //arxiv.org/abs/1003.3707, 2010.

[4] K. Gomadam, V. Cadambe, and S. Jafar, "A distributed numerical approach to interference alignment and applications to wireless interference networks," IEEE Trans. Inf. Theory, (to appear).

[5] H. Yu and Y. Sung, "Least squares approach to joint beam design for interference alignment in multiuser multi-input multi-output interference channels," IEEE Trans. Signal Process., vol. 58, no. 9, pp. 4960 -4966, 2010.

[6] O. E. Ayach and R. W. H. Jr., "Interference alignment with analog channel state feedback," http://arxiv.org/abs/1010.2787, 2010.

[7] H. Bolcskei and I. Thukral, "Interference alignment with limited feedback," in Proc. IEEE ISIT, 2009, pp. $1759-1763$.

[8] R. Krishnamachari and M. Varanasi, "Interference alignment under limited feedback for mimo interference channels," in Proc. IEEE ISIT, 2010, pp. $619-623$.

[9] D. Love, J. Heath, R.W., and T. Strohmer, "Grassmannian beamforming for multiple-input multiple-output wireless systems," IEEE Trans. Inf. Theory, vol. 49, no. 10, pp. 2735 - 2747, 2003.

[10] B. Hassibi and B. Hochwald, "How much training is needed in multipleantenna wireless links?" IEEE Trans. Inf. Theory, vol. 49, no. 4, pp. 951 $-963,2003$.

[11] L. Zheng and D. Tse, "Communication on the grassmann manifold: a geometric approach to the noncoherent multiple-antenna channel," IEEE Trans. Inf. Theory, vol. 48, no. 2, pp. 359 -383, Feb. 2002.

[12] N. Jindal, "Mimo broadcast channels with finite-rate feedback," IEEE Trans. Inf. Theory, vol. 52, no. 11, pp. 5045 -5060, 2006.

[13] D. Lee and D. Neuhoff, "Asymptotic distribution of the errors in scalar and vector quantizers," IEEE Trans. Inf. Theory, vol. 42, no. 2, pp. 446 -460, Mar. 1996.

[14] J. Zheng, E. Duni, and B. Rao, "Analysis of multiple-antenna systems with finite-rate feedback using high-resolution quantization theory," IEEE Trans. Signal Process., vol. 55, no. 4, pp. 1461 -1476, 2007.

[15] N. Ravindran and N. Jindal, "Multi-user diversity vs. accurate channel state information in mimo downlink channels," http://arxiv.org/abs/0907. 1099, 2009. 